EPiC Series in Engineering
Volume 3, 2018, Pages 1979-1988
HIC 2018. 13th International
Conference on Hydroinformatics

\title{
Centrality Metrics for Water Distribution Networks
}

\author{
Antonietta Simone ${ }^{1}$, Luca Ridolfi ${ }^{2}$, Daniele B. Laucelli ${ }^{1}$, Luigi Berardi ${ }^{*}$ \\ and Orazio Giustolisi ${ }^{1}$ \\ ${ }^{1}$ Politecnico di Bari, Via E. Orabona 4, 70125 Bari, Italy \\ ${ }^{2}$ Politecnico di Torino, C.so Duca degli Abruzzi, 2410129, Torino, Italy \\ antonietta.simone@poliba.it, luca.ridolfi@polito.it, \\ danielebiagio.laucelli@poliba.it, luigi.berardi@poliba.it, \\ orazio.giustolisiepoliba.it
}

\begin{abstract}
Complex Network Theory (CNT) studies theoretical and physical systems as networks, considering their features deriving from the internal connectivity between elements defined as vertex and links. In order to quantify the importance of these elements in real networked systems, researches proposed several centrality metrics.

The use of CNT centrality metrics for analysis, planning and management of infrastructure networks (streets, water systems, etc.), for example in terms of reliability and vulnerability, is today a relevant issue also considering their influences in socioeconomics and environmental matters. From CNT standpoint, water distribution networks (WDNs) are infrastructure networks that can be analyzed considering some peculiar features deriving from their spatial characteristics.

The paper focuses on CNT centrality metrics and proposes novel hydraulic centrality metrics useful for understanding the WDNs behavior. Furthermore, the study is intended to evaluate the feasibility of coupling hydraulic and topologic centrality metrics based on links, in order to obtain information that are more useful from the hydraulic point of view. This way, centrality metrics of the CNT become a complementary tool to hydraulic modelling for WDNs analysis and management.
\end{abstract}

\section{Introduction}

In Complex Network Theory, several centrality metrics have been proposed for describing the network structure of real systems. Among them, centrality metrics adopted for real networks (Borgatti, 2005) are: degree (Nieminen, 1974; Freeman, 1979), closeness (Freeman, 1979), betweenness

\footnotetext{
${ }^{*}$ Corresponding author: luigi.berardi@poliba.it
} 
(Freeman, 1977), eigenvector (Bonacich, 1972), Katz centrality (Katz, 1953), PageRank (Page , et al., (1998),), Hub and Authorities (Kleinberg J., 1999). Centrality metrics enable ranking or measuring the importance of network nodes (Freeman, 1979; Bonacich, 1987). Freeman (Freeman, 1979) introduced the concept of centralization, i.e., measuring the different level of importance of nodes in the network with respect to its specific behavior. Nonetheless, the choice of a metric instead of another can be not an easy decision since the features of the network under analysis (Benzi \& Klymko, 2015) should drive it, in general.

For spatial networks, centrality metrics based on the concept of shortest paths (e.g., closeness and betweenness) are relevant with respect to the flux of information (e.g., water flow in hydraulic systems). Water distribution networks (WDNs) are special constrained spatial networks, whose installation of pipes is constrained by buildings and streets. This fact limits the maximum nodal degree (Giustolisi, et al., 2017) and motivates the development of a novel metric, named neighborhood degree, representing the sum of the degrees of the adjacent nodes. Neighborhood degree provides a richer information about the centrality of the node in terms of local connectivity. Therefore, for WDNs, the extended neighborhood degree represents a local centrality metric of the nodal relevance, while betweenness and closeness centralities are global metrics since they are based on shortest paths between the entire set of couples of nodes.

For WDNs, hydraulic centrality metrics can be defined based on WDN hydraulic status (e.g., pipe flows, nodal pressures, leakages) given some boundary conditions, (e.g., costumer demands, water level in tanks, pumps and valve status, etc.). Differently from topological metrics, hydraulic metrics, are time varying being the WDN hydraulic behavior changing over time (Giustolisi \& Walski , 2012).

This contribution aims at describing topological and hydraulic centrality metrics as tools to support WDNs analysis and management. Their possible combinations are also investigated as a way to couple over time the connectivity structure (shortest paths) and the hydraulic behavior of the WDN.

\section{CNT centrality metrics for WDNs}

We here report some Centrality metrics in order to clarify the concept of "importance" within the network discussing the formulation of the betweenness, closeness and neighbourhood degree, as relevant centrality metrics for studying WDNs.

\section{Betweenness}

The betweenness (B) centrality by Freeman (Freeman, 1977; Freeman, 1979)measures the centrality in a graph based on shortest paths. Given a node $i$, and two nodes $s$ and $t$, a number $\sigma_{\mathrm{st}}(i)$ of shortest paths exist between $s$ and $t$ traversing node $i$. The sum of the fractions of the shortest paths between all couple of nodes $s$ and $t$ in the network is the betweenness centrality of node $i$, as in Eq. (1) (Freeman, 1979; Freeman, 1977; Anthonisse, 1971),

$$
C_{i}^{B}=\sum_{s \neq i \neq t \in V} \frac{\sigma_{s t}(i)}{\sigma_{s t}}
$$

where $\sigma_{\mathrm{st}}$ is the number of all shortest paths from node $s$ to node $t$ and $V$ is the set of vertices belonging to the graph $G$. The node with the highest betweenness centrality, i.e. traversed by the highest fraction of shortest paths, is the most "important".

\section{Closeness}

The closeness (C) centrality by Freeman (Freeman, 1979) allows measuring the internal distances among nodes. Given a node $i$, its closeness is based on the concept of shortest paths involving all other 
nodes $j$; assuming that $\sum d_{i j}$ is the sum of $i, j$ distances, the formulation of the closeness $(\mathrm{C})$ centrality is:

$$
C_{i}^{C}=\frac{1}{\sum_{j} d_{i j}}
$$

\section{Degree and Neighbourhood degree}

The degree centrality introduced by Niemen (Nieminen, 1974) represents the number of links connected to a node and describes the local connectivity of the network structure. As mentioned above, the neighbourhood nodal degree (N) (Giustolisi, et al., 2017) extends the concept of standard degree since it measures the nodal connectivity at different levels of neighbours. Assuming $A_{i j}$ as the elements of the network adjacency matrix, $k(j)$ the standard degree of the node $\mathrm{j}$ belonging to the topological neighborhood $N(i)$ (adjacent nodes), then the neighborhood degree $k_{n}(i)$ of node $i$, is formulated as:

$$
C_{i}^{N}=k_{n}(i)=\sum_{j \in N(i)} A_{i j} k(j)
$$

Since the neighborhood degree centrality measures the connectivity of the network structure involving a neighbor of nodes, it is effective to quantify the nodal importance in spatial networks like WDNs. The definition of neighborhood degree involving nodes at distance $n$ can be named $n$ neighborhood $(n-\mathrm{N})$ nodal degree and, consistently with the 1-neighborhood nodal degree (Giustolisi, et al., 2017), represents the number of links connected to any node having a distance lower or equal to $n$ from that node. Therefore, the $n$-neighborhood nodal degree centrality measures the connectivity of the network structure grouping nodes according to the assumed distance $n$.

\section{Tailoring centrality metrics for WDNs}

This study aims at exploring centrality metrics tailored for WDNs in order to consider the "importance" of pipes and not of nodes. In fact, in the case of WDNs, nodes are generally elements to transfer the water (information) while the water is delivered at pipe (link) level, e.g. water is supplied to connections distributed along pipes and leakages are assumed to flow out from pipes. Therefore, demand nodes are not significant for the connectivity structure of the WDNs because pipes (links) can fail while connection nodes generally do not fail. Moreover, hydraulic status variables of WDNs represent centrality metrics based on the direct graph generated by flows in the network. The shortest paths can be assumed as driven by the directions of the flows and there is the need of tailoring nodal centrality metrics in order to consider pipe (link) importance. In more detail we will consider herein the edge betweenness proposed by Girvan and Newman (Girvan \& Newman, 2002) and pipes closeness and $n$-neighbourhood degree centralities.

\section{Edge Betweenness}

It is similar to betweenness centrality, but refers to the generic link $l$. Let's assume that $\sigma_{\mathrm{st}}(l)$ is the number of shortest paths from node $s$ to node $t$ passing along the edge $l$ and $\sigma_{\mathrm{st}}$ is the number of all shortest paths from node $s$ to node $t$. The edge betweenness centrality (EB) of link $l$ the sum of the fractions $\sigma_{\mathrm{st}}(l) / \sigma_{\mathrm{st}}$ for all the couples of nodes $s, t$ in the network:

$$
C_{l}^{B}=\sum_{\substack{s \neq t \in V \\ l \in E}} \frac{\sigma_{s t}(l)}{\sigma_{s t}}
$$


where $V$ and $E$ are the set of vertexes (nodes) and edges (pipes) belonging to the network. The link with the highest edge betweenness centrality is the most important.

\section{Edge Closeness}

The edge closeness (EC) is similar to the closeness centrality (C) but it considers the distance between pipes, thus using the edge adjacency matrix (Simone, et al., 2016). Assuming that $\sum d_{l e}$ is the sum of distances of edges e of the network from $l, \mathrm{EC}$ is formulated as:

$$
C_{l}^{C}=\frac{1}{\sum_{e} d_{l e}}
$$

\section{Edge Degree and Edge Neighbor degree}

The edge degree centrality is the number of links connected to one of the two ending nodes of that link, i.e. the adjacent pipes. The edge $n$-neighborhood degree is the number of links having a distance lower or equal to $n$ from a given link.

The edge $n$-neighborhood degree ( $n$-EN) centrality measures the connectivity of the network structure grouping pipes according to the distance $n$. The concept of grouping pipes is similar to that of grouping nodes (Giustolisi, et al., 2017) but it considers the fact that failures may occur at pipe level. High values of $n$-EN identify the most connected portions of the network in terms of pipes.

\section{Hydraulic Centrality metrics in WDNs}

For the sake of presentation in this work, the hydraulic variables used here are flows $(\mathrm{F})$ and leakages (L). Flow (F) represent a global centrality metric, similarly to betweenness and closeness centrality metrics, being related to the concept of the shortest paths. In fact, WDN hydraulics allow identifying the main flow paths based on WDN network structure and local hydraulic capacity, i.e. dependent on diameters. Leakages (L) represents a local centrality metric, because it gives information on individual elements, i.e. depending on pipe deterioration and local pipe pressure.

Table 1 shows the "global" or "local" meaning for both hydraulic centrality metrics and topological centrality metrics reported in section 2 .

Table 1. Global and local topologic and hydraulic centrality metrics for WDNs

\begin{tabular}{lcc}
\hline & Global & Local \\
\hline Hydraulic metrics & Flow (F) & Leakages (L) \\
\hline \multirow{2}{*}{ Topologic metrics } & Betweenness (B) & $n$-Neighbourhood $(n$-N) \\
\hline
\end{tabular}

Actually, pipe flowrates, leakages and average pressures can be considered as edge centrality metrics, while nodal pressures and customer demands can be considered as nodal centrality metrics. Those variables can be related to the direct graph generated by flows in the network. Thus, "Global" and "local" meaning of hydraulic variables can be also related to edge centrality metrics as reported in Table 2.

Table 2. Global and local topologic and hydraulic edge centrality metrics for WDNs

\begin{tabular}{lcc}
\hline & Global & Local \\
\hline Hydraulic metrics & Flow (F) & Leakages (L) \\
\hline Topologic metrics & $\begin{array}{c}\text { Edge Betweenness (EB) } \\
\text { Edge Closeness (EC) }\end{array}$ & $n$-Edge Neighbourhood (n-EN) \\
\hline
\end{tabular}


The possible coupling between topological components, used for characterizing the connectivity structure of the network represented by an undirected graph, and the hydraulic components, represented by a directed graph, can provide additional elements to support WDN analysis and management actions. In fact, using topologic centrality metrics with "hydraulic centrality" metrics is a way to consider the connectivity structure of the hydraulic domain with the specific behavior of the system, which varies over time. In such context, it is important to use hydraulic metrics in a consistent way as in Tables 1 and 2, meaning that we need to distinguish hydraulic variables relating to flow paths (pipe velocity, flowrate, residential time, etc.) with respect to local variables (pipe average pressure, leakages, etc.). Therefore, this work first shows the topological centrality metrics for a small network and then discusses global centrality metrics obtained by coupling flows (F) with closeness (C), betweenness (B) and their tailored expressions at edge level (EC, EB), as well as local centrality metrics coupling leakages (L) with $n$-neighborhood nodal degree $(n$-N) and $n$-edge neighborhood degree ( $n$-EN).

\section{Case study}

The strategy for centrality metrics in WDNs is presented using Apulian network (Giustolisi, et al., 2008), a small network composed of 24 nodes, 34 pipes and 1 reservoir (at 36.4 s.l.m.) reported in Figure 1.

Figures 2 shows betweenness and closeness centralities for Apulian network. Node 6 (central dark blue node) has the maximum betweenness and closeness centrality values, i.e. it is the most efficient in spreading the information (closeness), because it is the closest to the other nodes, and it is the most traversed by the shortest paths (betweenness). It is worth noting that closeness highlights important groups of nodes because, if one node is important, also its nearest nodes are important, for the same reason. On the other hand, betweenness identifies important paths in the network.

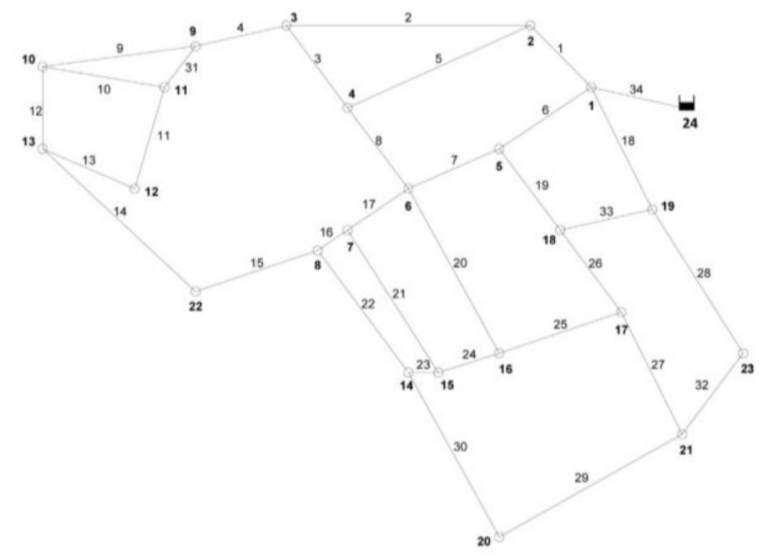

Figure 1. Apulian layout

Considering the inflow or the outflow in each node, also pipe flowrates can generate nodal centrality metrics. In order to analyze non-trivial scenarios for presenting and discussing the centrality metrics, a second reservoir (at 45 s.l.m.) was added at node 10, (see Figure 3), which results into a slightly more complex pattern of pipe flows. Since, the connectivity structure of the network is unchanged from the original and modifies Apulian layout, even after adding the second reservoir 2, betweenness and closeness centralities are the same as those in Figure 2. 


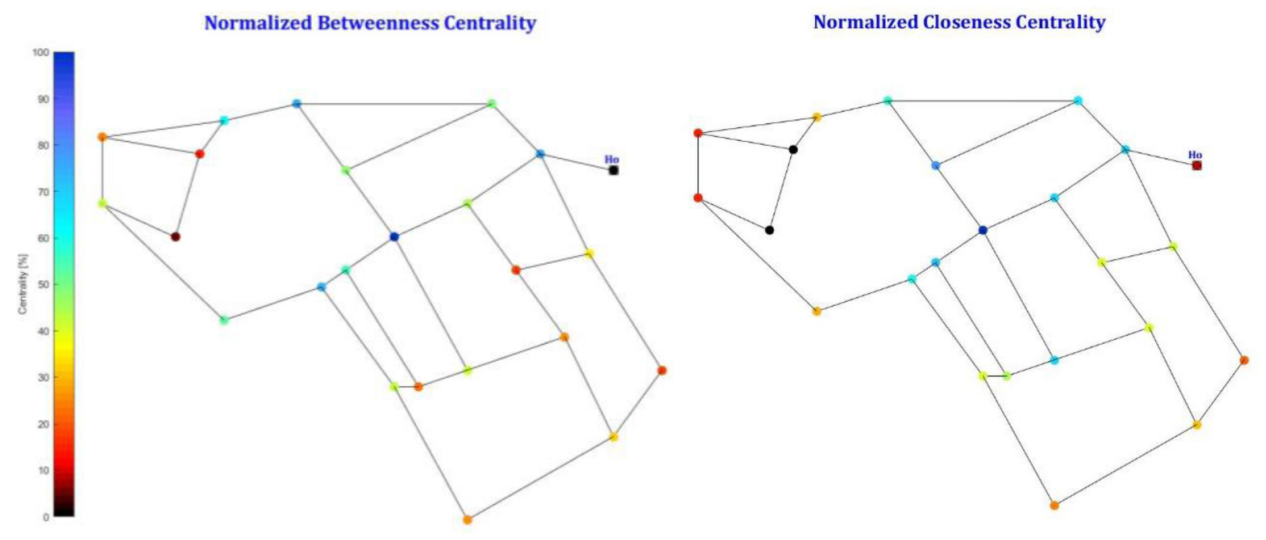

Figure 2. Betweenness (left) and closeness (right) centralities for Apulian network, normalized in the range $[0,100]$.

Figure 3 shows the flow centrality and it is evident that the nodes with the most significant values are close to the original reservoir 1 , although its water level is lower than reservoir 2 . This means that reservoirs and its closest nodes are the most important considering pipe flowrates. This is consistent with the fact that the network was originally designed to supply water from reservoir 1 only, thus pipes (diameters) linked to reservoir 2 are smaller than pipes linked to reservoir 1.

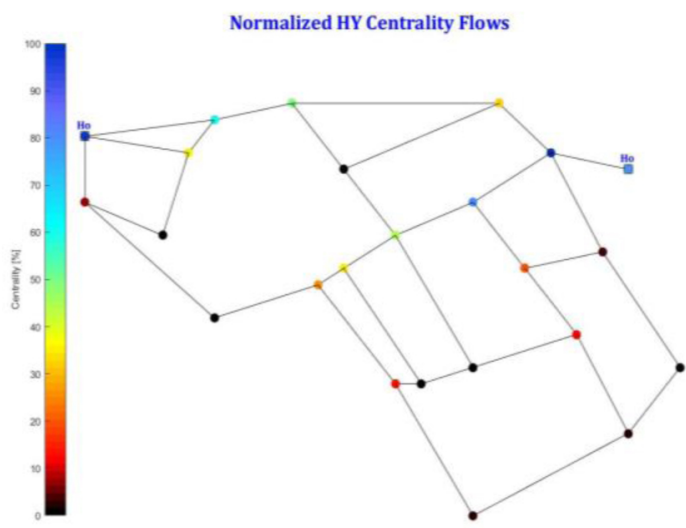

Figure 3. Flow centrality for modified Apulian network, normalized in the range [0, 100].

Figure 4 reports two "global" coupled centralities: flow (F) with betweenness (B) and flow (F) with closeness $(\mathrm{C})$. The coupled metrics show very similar preferential paths passing through the most important nodes, i.e. a path starting from node 24 , which cross nodes 1, 5, 6 and 7 to arrive at the node 8 and a path connecting two reservoirs that passes through nodes 9,3 and 2 .

Although such information is consistent with the hydraulic WDN behavior, such nodal metrics are not of direct relevance for WDN vulnerability analysis since they do not provide information about the most disruptive failure of pipes connected to that node. In fact, the nodal metrics assess the importance of nodes that are fictitious system components, although this is not technically sound because pipes are the network components actually failing. 

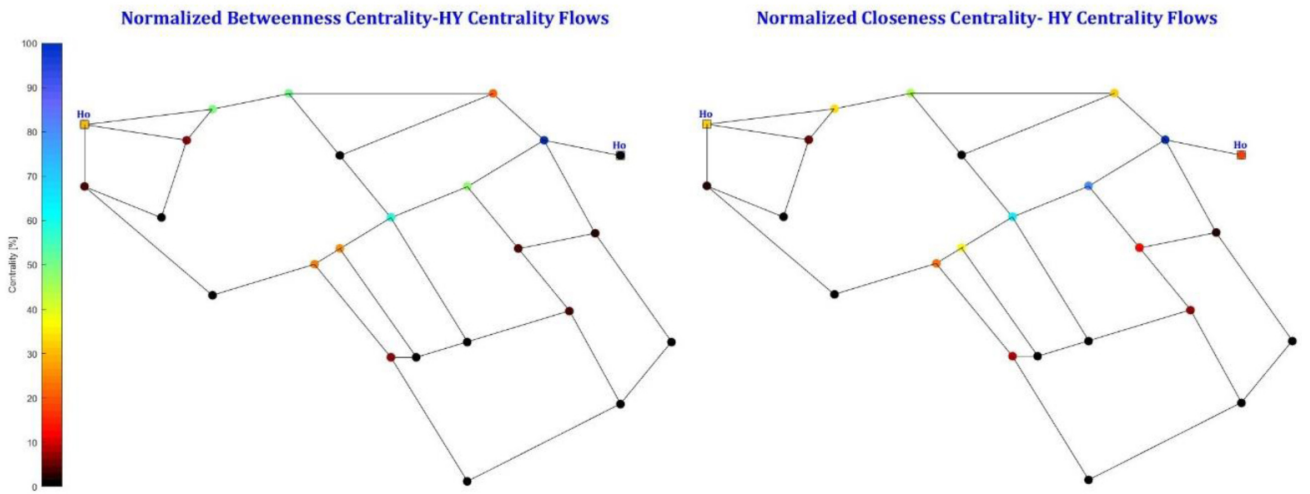

Figure 4. Coupling B-F (left) and C-F for Apulian network with two reservoirs.
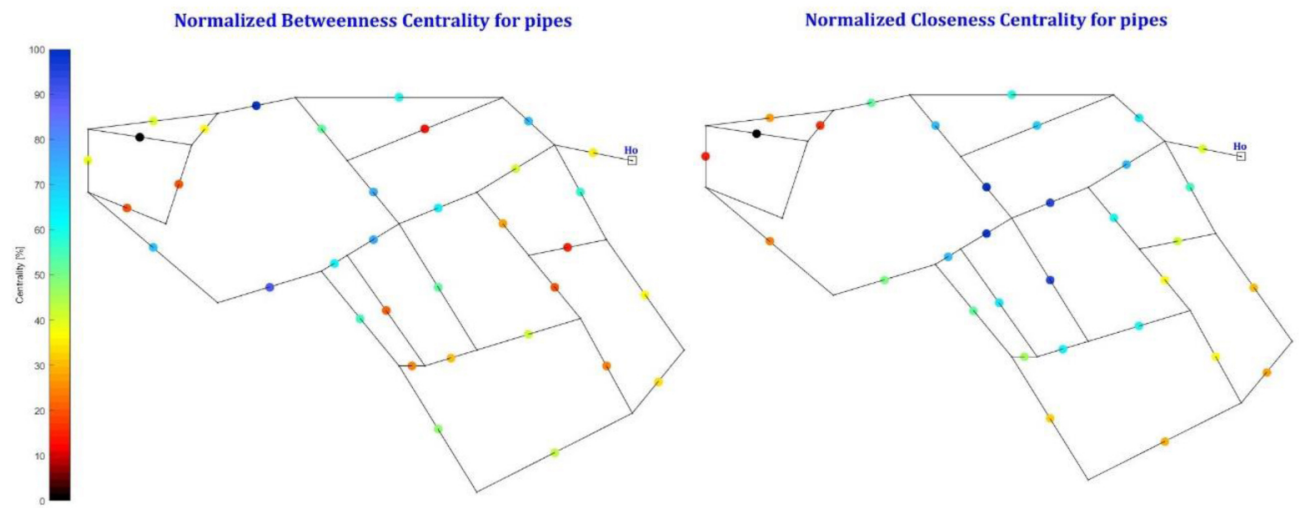

Figure 5. Edge betweenness (EB) (left) and edge closeness centrality (EC) (right) for Apulian network.

To obtain information that are most useful from the hydraulic point of view, let's consider topologic centrality metrics based on edge (i.e. Figures 5 and 6) and their coupling with hydraulic centrality metrics based on edge (i.e. Figure 7 and 8).

Similarly, to nodal centrality metrics, the topologic centrality metrics EB, EC, 1-EN and 2-EN in Figures 5 and 6 for the original Apulian WDN, hold for the modified Apulian WDN also.

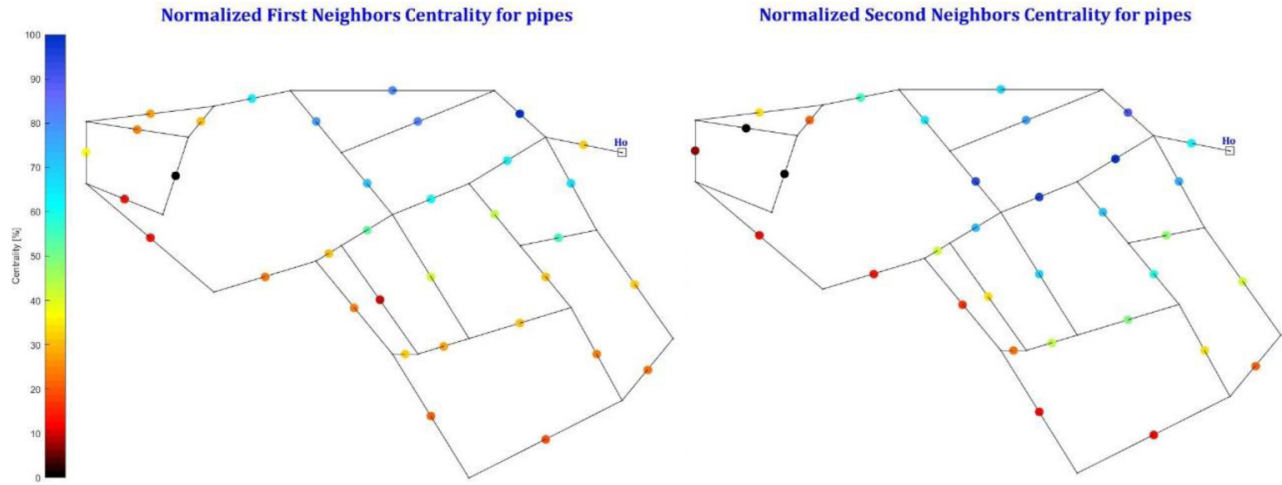

Figure 6. Edge 1-neighborhood degree (1-EN) (left) and 2-neighborhood degree (2-EN) (right). 
Figure 7 shows that coupling F with EB and with EC provides "global" information about the actual vulnerability of pipes 1, 6 and 18. The most critical is pipe 6 because it is the main pipe (i.e. the larger diameter) to transfer water from reservoir 1 to the hydraulic system. Moreover, Figure 7 shows that EB$\mathrm{F}$ and EC-F return similar preferential paths although the most important pipe does not correspond for two metrics. In fact, pipe 4, with the highest EB centrality (Figure 5-left) represents a strong connection between two main parts of a network, i.e. between many pairs of nodes through shortest paths. This happens because the EB carries information about the global connectivity of the network. Differently, pipe 6 with the highest EC, represents the most efficient pipe in spreading the water (information) in the network (i.e., the closest to the important pipes). In fact, EC gives information about the relevance of local connectivity to transfer water and coupling it with flows highlights the most important pipes to transfer water. From such perspective, pipes 6 is more important than pipe 34 because its failure would globally affect water transfer from both the reservoirs, while pipe 34 only for reservoir 1.

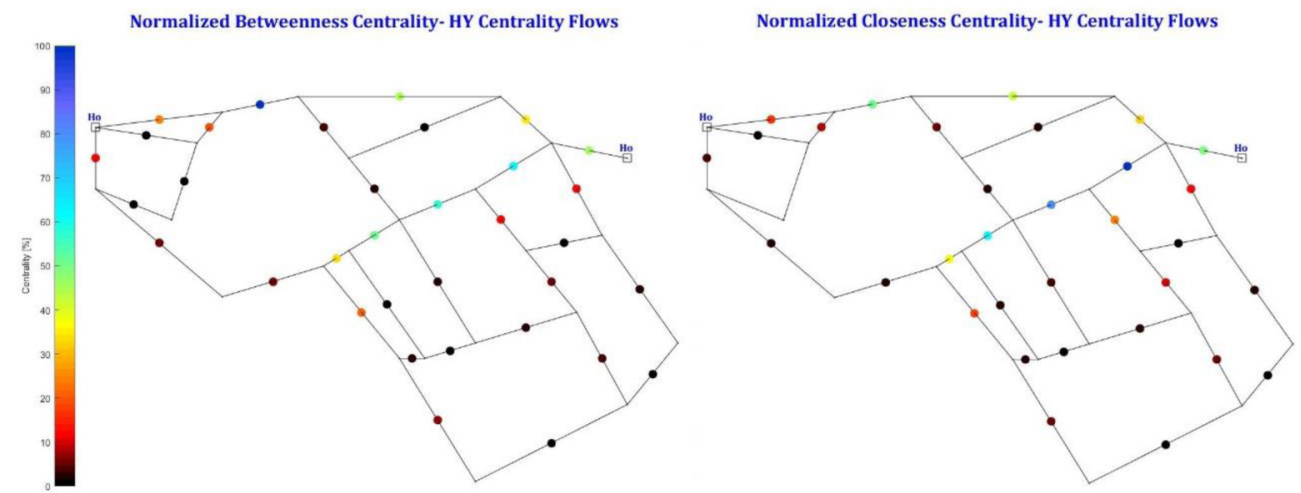

Figure 7. Coupling EB-F (left) and EC-F (right) for Apulian network with two reservoirs.

Figure 8 refers to the $\mathrm{n}$-EN that provides local information about the behavior of the main elements of the network, based on their connectivity, through the formation of groups of pipes of similar colors representing the most connected portions of the network.

The same figure shows the coupling of Leakages (L), which is relevant for leakage management actions, with $n$-EN. In more details, leakages in the modified Apulian WDN was computed using pressure driven modelling (Giustolisi, et al., 2008), thus reflect the joint effect of pipe deterioration and pressure.

The plots of 1EN-L and 2EN-L evidence leakages areas, which could be useful, e.g. for rehabilitation actions. This means that, for a larger network, the selection of the $n \mathrm{EN}-\mathrm{L}$ can group the leakages areas providing a more effective picture of the hydraulic system for leakage management. In particular, Figure 8 center (L-1EN) shows that leakages are concentrated at the upper part of the network, and this trend is strengthened by the figure on the right (L-2EN). 

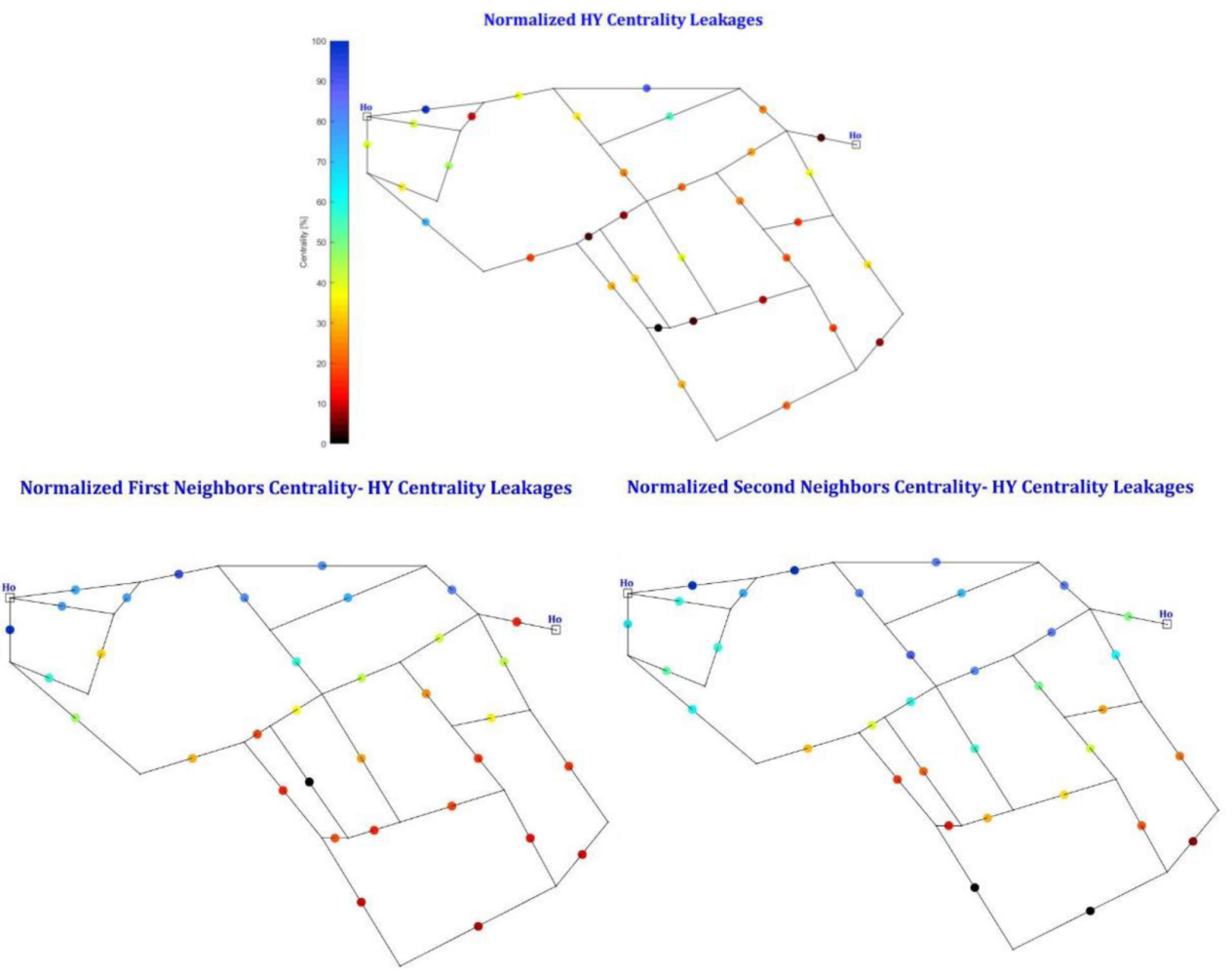

Figure 8. Leakages (L) (top), L-1EN (left-down) and L-2EN (right-down) centrality for modified Apulian WDN.

\section{Conclusions}

This contribution proposes the study of existing topological centrality metrics proposed by CNT and of some hydraulic centrality metrics for WDNs. In this perspective, the aim is to assess the feasibility and the benefits of coupling of hydraulic and topologic centrality metrics based on links, in order to obtain information that are most useful from the hydraulic point of view.

In order to discuss the features of centrality metrics for WDN analysis, the vulnerability point of view is undertaken although their use for other analysis and management purposes is an open issue. Different combinations of metrics are shown to provide information from different perspectives to support WDN management actions, in addition to accurate WDN modelling.

\section{Reference}

Anthonisse, J., 1971. The Rush in a Graph. Amsterdam: Mathematisch Centrum, s.n.

Benzi, M. \& Klymko, C., 2015. On the limiting behavior of parameter-dependent network centrality measures,. SIAM Journal on Matrix Analysis and Applications, 36(2), pp. 686-706.

Bonacich, P., 1972. Factoring and weighting approaches to status scores and clique identification. Journal of Mathematical Sociology, 2(1), pp. 113-120. 
Bonacich, P., 1987. Power and centrality: a family of measures. Amer. J. Sociology, 92(5), pp. 11701182.

Borgatti, S. P., 2005. Centrality and network flow. Social networks, 27(1), pp. 55-71.

Freeman, L., 1979. Centrality in social networks: Conceptual clarification. Social Networks, 1(3), pp. 215-239.

Freeman, L. C., 1977. A set of measures of centrality based on betweenness. Sociometry, 40(1), pp. $35-41$.

Girvan, M. \& Newman, M., 2002. Community structure in social and biological networks. Proc. Natl. Acad. Sci. USA, 99(12), pp. 7821-7826.

Giustolisi, O., Savic, D. \& Kapelan, Z., 2008. Pressure-driven demand and leakage simulation for water distribution networks. J. Hydraul. Eng., 134(5), pp. 626-635.

Giustolisi, O., Simone, A. \& Ridolfi, L., 2017. Network structure classification and features of water distribution systems. Water Resources Research, Issue 53, p. 3407-3423.

Giustolisi, O. \& Walski, T., 2012. Demand Components in Water Distribution Network Analysis. Journal of Water Resources Planning and Management, 138(4), pp. 356-367. $39-43$.

Katz, L., 1953. A new status index derived from socio metric analysis. Psychometrika, 18(1), pp.

Kleinberg J., M., 1999. Authoritative sources in a hyperlinked environment. Journal of the ACM, 46(5), pp. 604-632.

Nieminen, J., 1974. On centrality in a graph,. Scandinavian Journal of Psychology, 15(1), pp. 322336.

Page , L., Brin, S., Motwani, R. \& Winograd, T., (1998),. The PageRank Citation Ranking: Bringing Order to the Web. Brisbane, Australia., Proceedings of the 7th International World Wide Web Conference, p. 161-172.

Simone, A., Giustolisi, O. \& Laucelli, D. B., 2016. A proposal of optimal sampling design using a modularity strategy. Water Resources Research, 52(8), pp. 6171-6185. 